\title{
Somatotopy of placebo analgesia is independent of spatial attention
}

This article was published in the following Dove Press journal:

Journal of Pain Research

23 March 201I

Number of times this article has been viewed

\section{Claudia Domnick' \\ Jürgen Lorenz ${ }^{2}$ \\ Michael Hauck ${ }^{1,3}$ \\ 'Department of Neurophysiology and Pathophysiology, University \\ Medical Center Hamburg-Eppendorf, Hamburg, Germany; ${ }^{2}$ Faculty of Life \\ Sciences, Hamburg University of Applied Sciences, Hamburg, Germany; ${ }^{3}$ Department of Neurology, University Medical Center Hamburg-Eppendorf, Hamburg, Germany}

\begin{abstract}
Placebo analgesia is being increasingly appraised as an effective support of pharmacological and surgical treatments of pain. The understanding of its neurobiological and psychological basis is therefore of high clinical relevance. It has been shown that placebo analgesia is somatotopically organized and relies on endogenous opioids. However, it is not clear whether temporal fluctuations of cue-dependent spatial attention account for the site specificity of placebo analgesia or whether a somatotopic placebo effect is possible without an attentional focus on the respective location. To address this issue we induced placebo expectations for one specific foot in healthy subjects, the other foot serving as a control location. The feet were stimulated in random order by painful laser stimuli. Half of the pulses were cued for stimulus location, whereas in the other half of trials the subjects were naïve about the location. We found that about half of the subjects exhibited a somatotopic placebo effect that was statistically independent of the spatial cue. We suggest that, after the induction of an initial expectation, placebo analgesia is spatially specific but does not necessarily depend on momentary fluctuations of spatial attention. This result rather suggests that the somatotopy of placebo analgesia relies on the creation of spatially guided expectations or conditioning, but can be maintained without ongoing monitoring of the affected body part.
\end{abstract}

Keywords: placebo analgesia, spatial attention, somatotopy

\section{Introduction}

Placebo analgesia is the pain relieving effect of an intrinsically inactive substance which is caused by the patient's expectation of its effectiveness. ${ }^{1}$ Evidence led to the practical conclusion that placebo is no longer regarded a delusion of the patient but a potential to increase the effect of pharmacological or physical therapy. ${ }^{2}$ It has been shown that placebo analgesia strongly relies on prior experience and expectation of pain relief ${ }^{3,4}$ and has been linked to opioidergic and nonopioidergic mechanisms. ${ }^{5}$ Functional brain imaging studies revealed that placebo analgesia is indeed related to decreased activity in pain-related areas and increased activity in frontal regions including the anterior cingulate cortex. ${ }^{6-8}$

Placebo analgesia, and more generally endogenous analgesia, has traditionally been considered a phenomenon that affects the entire body without any site-specificity. However, Montgomery and Kirsch ${ }^{9}$ challenged this view by demonstrating that only pain in the body part that was expected to be treated responded to pain relief but not remote body areas. Later, Benedetti and colleagues ${ }^{10}$ showed that somatotopic manifestations of placebo relied on endogenous opiates. In addition, Watson et $\mathrm{al}^{11}$ who gave ambiguous information about the site of analgesia found a placebo effect that
Correspondence: Claudia Domnick Martinistrasse 52, 20246 Hamburg, Germany

Tel +49407410 56170

Fax +4940741057752

Email c.domnick@uke.uni-hamburg.de 
was specific for one arm in some of their subjects while other subjects showed a bilateral response. However, as in these studies the subjects could anticipate the stimulus location, it is possible that trial-by-trial variation of spatial attention and not the existence of somatotopically organized descending pathways could have accounted for the observations described above. Therefore, the key question of the present study was: does the site-specificity of placebo analgesia depend on momentary fluctuations of spatial attention, or is its initiation by spatially guided expectancies sufficient for it to be maintained in the absence of spatial attention.

To investigate this question, we applied an inert cream to both feet while suggesting that one foot would be treated by a potent analgesic. We induced placebo cognitions for one specific foot and manipulated spatial attention by presenting either a visual cue that announced the location of the painful laser stimulus or a visual stimulus that did not inform about the location of stimulus application. If momentary variation of spatial attention induced by informative cues was crucial for the somatotopic organization of placebo analgesia, pain relief would appear only during trials with an informative cue. Alternatively, if spatial attention was not necessary for a spatially specific placebo effect, placebo responders would perceive less pain regardless of the cue.

\section{Methods}

\section{Participants}

Twenty-eight healthy volunteers (15 male) were paid to participate in this study. They were aged 20 to 34 years (mean $24 \pm 3$ ). Written informed consent was obtained and the subjects were informed that they could terminate the experiment at any time. The study was conducted according to the Declaration of Helsinki and approved by the local ethics committee. After finishing the project, subjects were debriefed by an information letter about the real purpose of the experiment. One subject was excluded from the analysis because of problems with the instructions.

\section{Pain stimulus}

For stimulation of the dorsum of the feet a Thulium YAG laser (wavelength $2 \mu \mathrm{m}$, StarMedTec, Starnberg, Germany) was used. The stimuli were brief infrared laser pulses of 1 millisecond duration and a beam diameter of $5 \mathrm{~mm}$. Before the experiment, individual pain thresholds were determined for the two feet separately using 3 series of increasing and decreasing stimuli. Beginning at $160 \mathrm{~mJ}$ we used a step size of $20 \mathrm{~mJ}$. Pain was defined as a feeling of a light pinprick comparable to the pulling of a hair.

\section{Procedure}

The experimenter wore a white lab coat and informed the subjects that we were investigating the effect of a new analgesic cream containing lidocaine. In fact, an inactive cream was applied with a wooden applicator to the dorsa of both feet of the subjects while the experimenter wore surgical gloves. The subjects were told that the "analgesic" cream was applied to one particular foot, while the other foot was treated with an inactive cream as a control containing the same carrier substance without lidocaine. The site of placebo manipulation was further defined using a series of conditioning trials (see below) and counterbalanced across subjects.

During the experiment the subjects were seated in a comfortable chair in front of a computer screen. Their feet were positioned such that they were not able to see the location of the stimulus application. The design is illustrated in Figure 1. The experiment consisted of 2 conditioning blocks of 10 trials and 4 experimental blocks of 80 trials. At the beginning of each trial the subject was shown a visual cue for the stimulus location on the screen. Two seconds later the laser pulse was delivered followed by a visual analog scale on the screen 3 seconds later. The subjects had to rate stimulus intensity by completing the scale using a joystick during the next 1.5 seconds. The lower end of the scale $(0 \%)$ designated "no pain" while the upper end indicated "worst imaginable pain" (100\%). During the conditioning blocks the stimulus intensity delivered to the placebo foot was 1.5-fold pain threshold while the control site was stimulated with 2-fold threshold. Due to the fact that subjects were told that the applied intensity was the same for both feet, we expected placebo analgesia at the site where the subjects attributed the perception of lower intensity to a treatment effect. The two feet were stimulated in alternate order and in all trials the cue indicated the stimulation site. During the following 4 experimental blocks, the stimulus intensity was identical (2-fold threshold) at both sites and stimuli were delivered in a fixed random order to both feet. Furthermore, in half of the trials, the arrow contained information about the stimulation location. In the other half of the trials, the arrow pointed to the top and thus did not contain information about where the stimulus would be presented. The cue design was chosen such that the subjects directed their attention spatially to the placebo and control sites after an informative cue in comparison with trials where no spatial information was given.

\section{Statistical analysis}

To detect a placebo effect on the single subject level, $t$-tests were calculated to test for differences in the pain ratings between the placebo and the control condition. A significant 
A

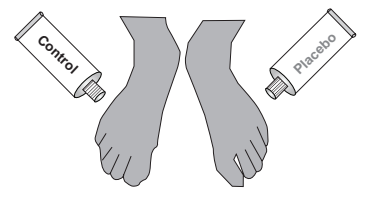

C
B

Conditioning blocks
Experimental blocks
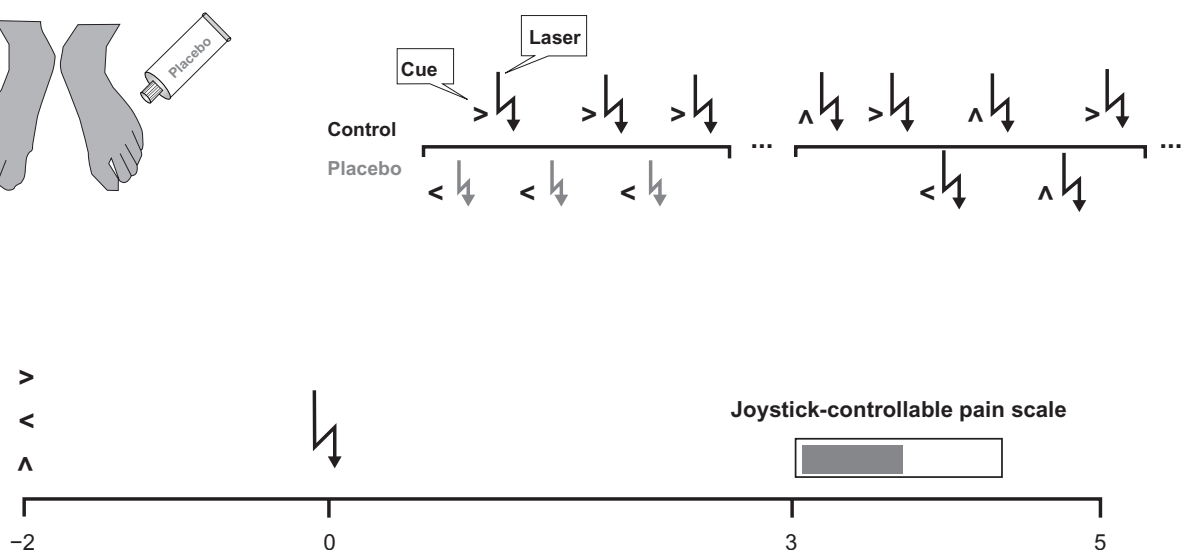

Figure I Experimental design. A) An inert cream was applied to both feet while subjects were told that one particular foot was treated by lidocaine. B) During the conditioning blocks, low intensity laser pulses were delivered to the placebo foot while the control foot was stimulated by high intensity pulses. All trials contained a cue about the stimulus location. During the experimental blocks, both feet were stimulated by high intensity laser pulses and only half of the trials contained information about the stimulus location. C) At the beginning of the trial, a visual cue (informative vs non-informative) preceded the laser stimulus that had to be rated on a visual analog scale 3 seconds later.

reduction in pain ratings in the placebo condition (critical $P$-value $=0.05)$ would indicate a placebo effect and subjects were classified as placebo responders. Subjects who did not exhibit any pain relief by the placebo treatment were classified as non-responders. The groups were compared for their pain ratings, thresholds, and age by nonparametric Mann-Whitney test. To make sure that there was a true overall placebo effect, a repeated-measures ANOVA was first calculated for the whole group including only the factor treatment (placebo, control). As we were specifically interested in the behavior of the placebo responders and its dependence on spatial attention, a repeated-measures ANOVA for the responder group was calculated including the within-subject factors treatment (placebo, control) and cue (informative, non-informative). Beforehand, normal distribution of all variables included in the ANOVA was confirmed by Kolmogorov-Smirnov test.

\section{Results}

\section{Single-subject statistics}

Of the 27 subjects, 13 were classified as placebo responders (48\%), as their pain ratings in the placebo condition were significantly lower than in the control condition $(P$-values ranging from $<0.01$ to 0.04$)$. Fourteen subjects $(52 \%)$ did not show any significant relief due to the treatment and thus were classified as non-responders.

\section{Group statistics}

Placebo responders did not differ from non-responders in age, pain threshold at the placebo and control foot, and ratings in the control condition.
The repeated-measures ANOVA revealed a significant treatment effect $(F(1,25)=11, P<0.005)$ due to lower ratings in the placebo condition $(38 \pm 15)$ than in the control condition $(41 \pm 15)$. As expected, the separate analysis for the responder group also resulted in a significant treatment effect $(F(1,12)=67, P<0.0001$; Figure 2$)$. In the placebo condition, the responders felt significantly less pain than in the control condition $(32 \pm 12$ vs $42 \pm 14)$. The effect of cue was not significant. Furthermore, the interaction between cue and treatment did not reach significance, indicating that the treatment effect did not depend on spatial attention.

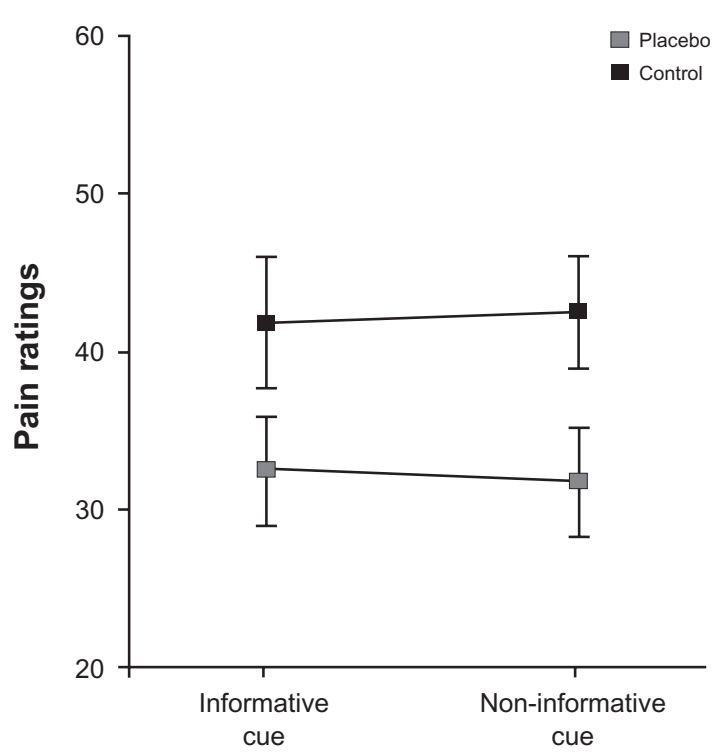

Figure 2 Group statistic for the placebo responders. Pain ratings in the placebo condition are significantly lower than in the control condition. Note that the relief by the placebo treatment is independent of the type of cue. 


\section{Discussion}

This study attempted to clarify whether momentary fluctuations of pain suppression by cue-induced expectations can explain the site-specificity of placebo analgesia. We treated the feet of our subjects by a placebo and a control cream. Conditioning trials in which the subjects attributed a difference of applied laser stimulus intensity to a pretended treatment effect served to induce placebo cognitions. About half of the subjects were placebo responders in subsequent experimental blocks of identical stimulus intensities applied to both feet. Our results support earlier findings of Montgomery and Kirsch ${ }^{9}$ and Benedetti et al ${ }^{10}$ that placebo analgesia is somatotopically organized. Furthermore, the major result of our study was that spatially informative and non-informative visual cues failed to affect the somatotopy of the placebo effect.

We induced top-down expectations of analgesia at the beginning of the experiment using verbal suggestion and conditioning. This has been reported useful in former studies $^{3,4}$ and to rely on opioid mechanisms. ${ }^{5}$ Verbal suggestion seems to be crucial for the spatial specificity of placebo analgesia, as Watson et a ${ }^{11}$ did not inform their subjects about the treatment site, resulting in a group of bilateral placebo responders. Our design does not allow ruling out that part of our non-responders exhibited a bilateral pain decrease. However, this seems unlikely, as the non-responders did not differ from the responders in their ratings in the control condition, ie, they gave high pain ratings in both treatment conditions. Our procedure might additionally have contributed to the idea that even very short painful laser stimuli can be sensitive to placebo analgesia, although other authors argued that placebo acts only on long-lasting pain perception. ${ }^{12,13}$

The absence of an interaction with cue-dependent spatial attention suggests indirectly a somatotopic organization of a subcortical route of pain modulation that interacts with spatially guided expectations or conditioning underlying the site specificity of placebo analgesia. It is known that brainstem structures such as the periaquaeductal grey (PAG) are involved in descending inhibition of pain at the dorsal horn level (for a review see ${ }^{14}$ ). PAG stimulation has been shown to induce analgesia for several weeks that was naloxone reversible, and thus depended on opioid mechanisms. ${ }^{15}$ Furthermore, a rough somatotopy has been demonstrated that affected distinct extremities ${ }^{16}$ and upper versus lower body parts ${ }^{17}$ differently. Therefore, placebo analgesia that is specific for one foot and lasts throughout an experiment of 1 hour can possibly be mediated by this structure.

Functional brain imaging studies demonstrated the involvement of prefrontal networks during anticipation and processing of pain stimuli after the induction of placebo analgesia. ${ }^{6,7}$ These studies showed that activity in frontal regions such as the anterior cingulate gyrus ${ }^{7}$ and the prefrontal/orbitofrontal cortex, ${ }^{6}$ engaged during attention and expectation, was related to placebo analgesia, and correlated with increased activity in the brainstem PAG. This fits with findings that frontal regions and midbrain structures share common opioidergic mechanisms related to placebo analgesia. ${ }^{18,19}$ Wager et $l^{6}$ and Bingel and colleagues ${ }^{7}$ demonstrated by event-related functional magnetic resonance imaging during cues of pain stimulation at placebo and control sites that frontal regions, such as the dorsolateral prefrontal cortex (DLPFC), processing top-down information about the stimulus, initiate the descending inhibition by the PAG in a phasic manner. Recently, low-frequency repetitive transcranial magnetic stimulation of the DLPFC has been found to completely block placebo analgesia. ${ }^{20,21}$ One might therefore expect - according to the alternative hypothesis of our study - that a phasic mode of spatial attention can account for the site specificity of placebo analgesia. In their elegantly designed study, Benedetti et $\mathrm{a}^{10}$ applied a tonic pain stimulus simultaneously to both feet and hands using subcutaneous injections of capsaicin via a computercontrolled injection pump. One or two of these sites were treated by a placebo cream. Electrical pulses announced the location at which pain had to be rated by the subjects. These cues occurred every 7 seconds. The spatial specificity of placebo analgesia that the authors observed could therefore be the result of a temporal fluctuation of pain suppression by expectancy between successive cues, governed by the DLPFC, without being necessarily dependent on a somatotopic organization of the descending pathway. However, although our results are compatible with the assumption that the DLPFC is important to engage endogenous inhibition during placebo analgesia, the somatotopy of the network is likely represented in different areas, probably primary and secondary somatosensory cortices and subcortical areas such as the brainstem.

The absence of any cue effect can be considered a weakness of our study. Attention in general and cuing in particular has been repeatedly found to affect pain perception..$^{22-24}$ As we did not find an effect of the visual cue on pain perception one can argue that we cannot be sure about the effectiveness of our attentional manipulation. However, the typical amplification of pain by a valid cue would contradict the placebo effect and the two effects would cancel each other out. Therefore, one could not expect to find a main effect of the cue but only an interaction between 
the placebo treatment and spatial attention for which we had been looking.

\section{Conclusion}

As we did not observe a dependence of spatially specific placebo analgesia on a spatial cue, we conclude that somatotopically organized placebo analgesia is possible without spatial attention. We suggest that a spatially specific expectation of analgesia that is induced in the beginning is sufficient to recruit the frontal attention network. In turn, this network initiates the endogenous antinociceptive system, especially the brainstem PAG, which maintains the spatial information for a longer time by means of its own somatotopic organization. Therefore, a trial-by-trial fluctuation of spatial attention is not necessary.

\section{Acknowledgments}

Claudia Domnick and Michael Hauck were supported by UKE Junior Research Grant FFM F-161-1, by the European Commission (NEST-043457 "Mindbridge") and by the German Research Foundation (SE 1859/1-1). We thank Andreas K Engel for making this work possible, Hanna Krause, Marion Höfle, Nicole David and Till Schneider for helpful discussion, Christian Bähnisch and Roger Zimmermann for programming the stimulation, Christiane Reißmann and Kriemhild Saha for help during the experiment and Sabine Hauck for a final correction of the paper.

\section{Disclosure}

The authors declare no conflicts of interest.

\section{References}

1. Fields HL, Price DD. Placebo analgesia. In: McMahon SB, Koltzenburg M, editors. Textbook of Pain. Philadelphia: Churchill Livingstone; 2006: 361-367.

2. Oeltjenbruns J, Schafer M. Clinical significance of the placebo effect. Anaesthesist. 2008;57(5):447-463.

3. Price DD, Milling LS, Kirsch I, Duff A, Montgomery GH, Nicholls SS. An analysis of factors that contribute to the magnitude of placebo analgesia in an experimental paradigm. Pain. 1999;83(2): $147-156$.

4. Voudouris NJ, Peck CL, Coleman G. The role of conditioning and verbal expectancy in the placebo response. Pain. 1990;43(1):121-128.

Journal of Pain Research

\section{Publish your work in this journal}

The Journal of Pain Research is an international, peer-reviewed, open access, online journal that welcomes laboratory and clinical findings in the fields of pain research and the prevention and management of pain. Original research, reviews, symposium reports, hypothesis formation and commentaries are all considered for publication.
5. Amanzio M, Benedetti F. Neuropharmacological dissection of placebo analgesia: expectation-activated opioid systems versus conditioningactivated specific subsystems. J Neurosci. 1999;19(1):484-494.

6. Wager TD, Rilling JK, Smith EE, et al. Placebo-induced changes in FMRI in the anticipation and experience of pain. Science. 2004; 303(5661):1162-1167.

7. Bingel U, Lorenz J, Schoell E, Weiller C, Buchel C. Mechanisms of placebo analgesia: $\mathrm{rACC}$ recruitment of a subcortical antinociceptive network. Pain. 2006;120(1-2):8-15.

8. Nemoto H, Nemoto Y, Toda H, Mikuni M, Fukuyama H. Placebo analgesia: a PET study. Exp Brain Res. 2007;179(4):655-664.

9. Montgomery G, Kirsch I. Mechanisms of placebo pain reduction: an empirical investigation. Psychol Sci. 1996;7(3):174-176.

10. Benedetti F, Arduino C, Amanzio M. Somatotopic activation of opioid systems by target-directed expectations of analgesia. J Neurosci. 1999; 19(9):3639-3648.

11. Watson A, El-Deredy W, Bentley DE, Vogt BA, Jones AK. Categories of placebo response in the absence of site-specific expectation of analgesia. Pain. 2006;126(1-3):115-122.

12. Price DD, Fields HL. The contribution of desire and expectation to placebo analgesia: implications for new research strategies. In: A H, editors The Placebo Effect: An Interdisciplinary Exploration. Cambridge, MA: Harvard UP; 1997:117-137.

13. Price DD. Psychological and Neural Mechanisms of Pain. New York: Raven; 1988.

14. Basbaum AI, Fields HL. Endogenous pain control systems: brainstem spinal pathways and endorphin circuitry. Annu Rev Neurosci. 1984; 7: 309-338.

15. Baskin DS, Mehler WR, Hosobuchi Y, Richardson DE, Adams JE, Flitter MA. Autopsy analysis of the safety, efficacy and cartography of electrical stimulation of the central gray in humans. Brain Res. 1986; 371(2):231-236.

16. Soper WY, Melzack R. Stimulation-produced analgesia: evidence for somatotopic organization in the midbrain. Brain Res. 1982;251(2): 301-311.

17. Kasman GS, Rosenfeld JP. Opiate microinjections into midbrain do not affect the aversiveness of caudal trigeminal stimulation but produce somatotopically organized peripheral hypoalgesia. Brain Res. 1986; 383(1-2):271-278.

18. Wager TD, Scott DJ, Zubieta JK. Placebo effects on human muopioid activity during pain. Proc Natl Acad Sci U S A. 2007;104(26): 11056-11061.

19. Petrovic P, Kalso E, Petersson KM, Ingvar M. Placebo and opioid analgesia - imaging a shared neuronal network. Science. 2002;295(5560): 1737-1740.

20. Benedetti F. No prefrontal control, no placebo response. Pain. 148(3):357-358.

21. Krummenacher P, Candia V, Folkers G, Schedlowski M, Schonbachler G. Prefrontal cortex modulates placebo analgesia. Pain. 148(3):368-374.

22. Miron D, Duncan GH, Bushnell MC. Effects of attention on the intensity and unpleasantness of thermal pain. Pain. 1989;39(3):345-352.

23. Dowman R. Attentional set effects on spinal and supraspinal responses to pain. Psychophysiology. 2001;38(3):451-464.

24. Dowman R. Distraction produces an increase in pain-evoked anterior cingulate activity. Psychophysiology. 2004;41(4):613-624.

\section{Dovepress}

The manuscript management system is completely online and includes a very quick and fair peer-review system, which is all easy to use. Visit http://www.dovepress.com/testimonials.php to read real quotes from published authors. 